\title{
Estrés en la comunidad íctica en la pesca de arrastre del camarón en el norte del Golfo de California
}

\author{
Eloísa Herrera-Valdivia ${ }^{1,2}$, Juana López-Martínez ${ }^{1 *} \&$ Sergio Castillo Vargasmachuca ${ }^{2}$ \\ 1. Centro de Investigaciones Biológicas del Noroeste, S.C. Km 2.35 Carretera a Las Tinajas, S/N Colonia Tinajas. \\ Guaymas, Sonora, México. C. P. 85460; jlopez04@cibnor.mx \\ 2. Universidad Autónoma de Nayarit. Posgrado en Ciencias Biológico Agropecuarias, Carretera Tepic-Compostela Km. \\ 9, Xalisco Nayarit, C.P.63780, México; eloisa04@cibnor.mx, sergio_machuca_@hotmail.com \\ * Correspondencia
}

Recibido 16-IX-2014. Corregido 10-II-2015. Aceptado 09-III-2015.

\begin{abstract}
Determination of stress in fish community obtained from shrimp trawl fishing in Northern Gulf of California. Bottom trawling has been considered a fishing activity that affects and modifies habitats, because of its impacts in species composition and abundance, and the alteration in the structure and function of the ecosystem, that generates biodiversity loss. The Northern part of the Gulf of California has been considered a mega diverse zone with high endemism, and it is of growing interest by the international scientific community. In order to assess its potential changes in the fish community components of shrimp by-catch (FAC) in this area, a total of 119 trawls from 13 fishing boats were analyzed in Puerto Peñasco, based on 14 commercial fishing trips made within 9-90 m depth from 2010-2011. A random sample of $20 \mathrm{~kg}$ was obtained from each trawl, and was analyzed in the laboratory for species composition. In addition to the Index of Biological Value (IVB), Shannon diversity $\left(H^{\prime}\right)$, and Pielou evenness (J'), comparative abundance-biomass curves (ABC) were also estimated. Eucinostomus dowii showed the highest IVB $=480.25$; Porichthys analis showed greater relative abundance; and Pomadasys panamensis showed greater frequency of occurrence. The mean monthly values in diversity $H^{\prime}$ $=3.05\left(2.72>H^{\prime}<3.25\right)$ and $J^{\prime}=0.71\left(0.66<J^{\prime}>0.81\right)$ showed a tendency to decrease as the fishing season progressed. The comparative abundance-biomass curves (ABC), and the value of statistical $W$ showed moderate stress levels in March $(W=-0.022)$ and September $(W=-0.02) 2010$, and January $2011(W=-0.042)$. In conclusion, the Northern Gulf of California showed a well-structured community with a degree of moderate fishing stress. Rev. Biol. Trop. 63 (3): 741-754. Epub 2015 September 01.
\end{abstract}

Key words: Northern Gulf of California, trawl fisheries by-catch, curves ABC, community, stress.

A nivel internacional se ha dicho que la pesca efectuada con redes de arrastre ha sido objeto de señalamientos como causante de alteraciones químicas y biológicas en los fondos marinos (Dernie, Kaiser, Richardson, \& Warwick, 2003; Nieto, 2010; Pérez, 2011). Este tipo de pesca es selectiva y ha sido señalada por capturar especies de peces, crustáceos y moluscos no objetivos de la pesquería, con potenciales efectos adversos a sus poblaciones (Thrush \& Dayton, 2002; Rábago, López, Valdez, \& Nevárez, 2011). Esto provoca cambios en el funcionamiento del ecosistema (Caddy, 2007) y afectación sobre los depredadores (Myers \& Worm, 2003). Incluso se ha sugerido que puede colapsar poblaciones de especies de baja tasa de natalidad, como sucede con tiburones y rayas (Márquez, 2002; Morales, 2011).

El Golfo de California (GC) es uno de los ecosistemas marinos de mayor productividad en el mundo y con gran diversidad de especies asociado a la estabilidad del ecosistema, además de la gran variedad topográfica y batimétrica, que proporciona un mayor número de 
hábitats para peces y otros organismos marinos (López, Hernández, Herrera, Rábago, \& Morales, 2007).

El Norte del Golfo de California (NGC) posee al menos $7200 \mathrm{~km}^{2}$ (Nelson Ramírez, Arenas, Jacquemine, \& Prado, 1980) desde el Delta del Río Colorado hacia la región de las grandes islas: Isla Tiburón y Ángel de la Guardia, con una profundidad promedio de 200 $\mathrm{m}$, de aguas ricas en nutrientes y alta productividad en todo el año (Lluch \& Arias, 2000). Es considerado también como uno de los ecosistemas marinos más ricos del mundo (Cudney \& Turk, 1998), altamente dinámico y cambiante, lo que hace posible sostener grandes cadenas alimenticias debido al gran potencial alimenticio autotrófico. Más al norte se encuentra la Reserva de la Biosfera Alto Golfo de California y Delta del Río Colorado (RB) decretada en junio de 1993 (Diario Oficial de la Federación, 1993), de la cual $82 \%$ constituye la zona de amortiguamiento, lugar donde existe una gran actividad pesquera multiespecífica.

En el NGC la pesquería de camarones peneidos Litopenaeus stylirostris y Farfantepenaeus californiensis, genera una fuente importante de ingresos en la comunidad, cerca de $77 \%$ de la población vive de la pesca (López, Herrera, Rodríguez, \& Hernández, 2010). Esta actividad se desarrolla entre los 9 a $90 \mathrm{~m}$ de profundidad, donde opera la flota de arrastre y donde se localiza gran variedad de especies que forman parte de la fauna acompañante del camarón (FAC) compuesta de peces, crustáceos y moluscos (López, Rodríguez, Hernández, \& Herrera, 2011).

Con respecto a la comunidad íctica del GC, sobresalen los listados sistemáticos de peces en sistemas lagunares (Rodríguez, Abitia, Galván, \& Chávez, 1994), y en otros estudios, se incluyen aspectos de estructura, diversidad, abundancia y variaciones espacio-temporales en comunidades de peces (López et al., 2010). Si bien es cierto, los estudios basados en índices ecológicos nos aproximan a conocer la salud de las comunidades, Jennings y Reynolds (2000) sugieren que la utilización de estos no ha sido suficiente para evaluar impactos provocados por las redes de arrastre en las comunidades béntico demersales, debido a que no han mostrado cambios significativos. Esto hace necesario desarrollar o aplicar otros métodos tales como el uso de las curvas k-dominancia, como una herramienta más recomendable para identificar los niveles de estrés en que se encuentran las comunidades de peces (Jouffre $\&$ Inejih, 2005).

A pesar de la importancia pesquera y ecológica del NGC, existe escasa información sobre la ecología de comunidades de peces sujetas a la pesca de arrastre. En cuanto a análisis con agregados comunitarios como curvas $k$ dominancia (Lambshead, Plat, \& Shaud, 1983), y aplicaciones de la curva comparativa de Abundancia-Biomasa ABC (Clarke \& Warwick, 2001), este estudio representa la primera aproximación para detectar grados de alteración en la comunidad de peces (predicha como cambios en la comunidad por la intensificación de la pesca), donde especies pequeñas de rápido crecimiento y oportunistas se convierten en dominantes, siguiendo a Yemane, Field y Leslie (2005). Por todo anterior, el objetivo de esta investigación fue analizar el grado de estrés, de la comunidad de peces asociados a los fondos blandos, provocados por la pesca de arrastre de camarón en una zona megadiversa como lo es el NGC, con base en la utilización de estos análisis.

\section{MATERIALES Y MÉTODOS}

La composición de la FAC de Puerto Peñasco, Sonora, fue evaluada en 119 muestras obtenidas a bordo de la flota camaronera, durante las temporadas de pesca 2009-2010 y 2010-2011. Para esto, se integró un programa de cuatro observadores a bordo de la flota comercial, y adicionalmente, se contó con información de un crucero de veda del camarón recopilada en la misma zona, por el Instituto Nacional de Pesca (INAPESCA) en agosto 2011.

Los 119 arrastres de los 14 viajes fueron realizados en los diferentes caladeros de pesca del NGC (Fig. 1). El tiempo de arrastre varió de 


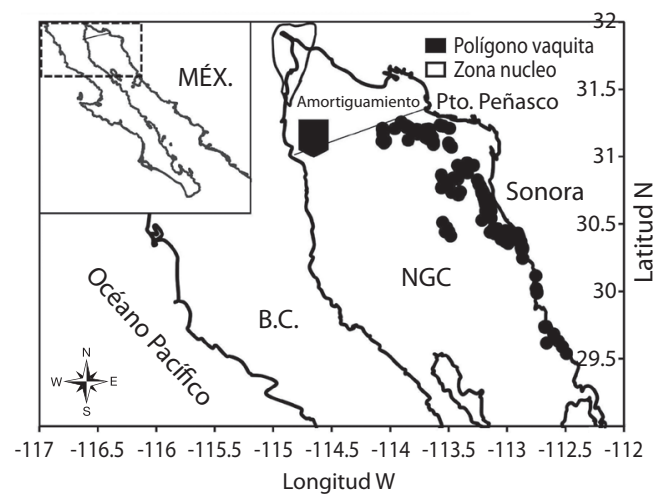

Fig. 1. Área de pesca y recolecta de muestras en el norte Golfo de California durante 2010-2011.

Fig. 1. Fishing and sample collection study area in the Northern Gulf of California from 2010-2011.

una a tres horas, con una velocidad promedio de $5.5 \mathrm{~km} /$ hora y profundidades entre $9-90 \mathrm{~m}$. Se utilizaron red de arrastre de relinga superior a $30.48 \mathrm{~m}$, relinga inferior a $38.1 \mathrm{~m}$, el peso de la cadena espantadora fue variable $50-75 \mathrm{~kg}$. La parte delantera con luz de malla de 140-160 $\mathrm{mm}$, cuerpo con malla de $120 \mathrm{~mm}$, la antebolsa de malla $100 \mathrm{~mm}$ y bolsa con luz de malla de $90 \mathrm{~mm}$.

La duración de los viajes fue de 4 a 15 días, en la bitácora de viaje se registraron los datos propios del lance (fecha, lance, profundidad, área de pesca, velocidad de arrastre, hora inicial-hora final, posición inicial y final del lance, peso y composición de la captura objetivo, captura total de FAC).

Para los análisis, se obtuvieron muestras al azar de aproximadamente $20 \mathrm{~kg}$ de la FAC. El tamaño de muestra se determinó siguiendo los criterios estándar (Box, Stuart, \& Hunter, 2008). La muestra fue etiquetada y guardada en bolsas de plástico y conservadas en la bodega del barco y posteriormente en bodegas frigoríficas de plantas pesqueras hasta su traslado al laboratorio de Pesquerías del Campus Guaymas del CIBNOR-Unidad Sonora.

Para la identificación de las especies, se utilizaron las claves de Jordan y Evermann (1893-1900), Norman (1934), Curran (1942), Zahuranec (1967), Miller y Lea (1972),
Castro-Aguirre (1978), Compagno (1984), Fischer et al. (1995), y con bibliografía especializada para algunos grupos: Castro-Aguirre y Espinosa-Pérez (1996) en rayas, McPhail (1958) para la familia Sciaenidae, Ginsburg (1958) en Pleuronectiformes, Rosenblatt y Johnson (1974) para Diplectrum, Walker y Rosenblatt (1988) para Porichthys.

Los nombres comunes fueron tomados de Love, Yoklavich y Thorsteinson (2002) y Robertson y Allen (2002).

Composición de especies: Se integró el listado de las 30 especies de peces bento-demersales más frecuentemente capturadas por la red de arrastre de la flota camaronera del norte del Golfo de California en el periodo estudiado. Esta información y sus abundancias, sirvió para obtener la estructura de las comunidades a través de los índices ecológicos aplicados.

Índice de Valor Biológico: \{XE TM6.2.4 Índice de valor biológico (IVB)" $\}$. El índice de Valor Biológico (IVB) de Sanders (1960) descrito Loya y Escofet (1990), tiene la ventaja de combinar la propiedad de abundancia relativa con la constancia de especies y es utilizada para estimar las especies de mayor importancia biológica en la FAC; así las especies que sean más constantes durante los muestreos serán más importantes que aquellas que presenten abundancias altas en menor número de muestreos, este índice se expresa de la siguiente manera

$$
I V B=\sum_{i j}^{j} \text { puntoij }
$$

donde: $I V B$ es el Índice de valor biológico, $\boldsymbol{i}$ corresponde a la abundancia relativa de cada especie y $j$ a las estaciones de recolección. Para medir los índices ecológicos de diversidad y riqueza específica se utilizó:

Diversidad de Shannon-Wiener (H'): se expresa de la siguiente manera:

$$
H^{\prime}=\sum_{i=1}^{n}\left(P_{i} \ln P_{i}\right)
$$


donde: $H^{\prime}$ = Índice de diversidad de Shannon y Weaver (1963), $P_{i}=$ Cociente del número de organismos de la especie $i$ entre el número total de la muestra, ni/NT.

Equitabilidad o Uniformidad J' (Pielou, 1966): J' $=H^{\prime} / \ln S$ donde $S=$ Se considera el número total de especies $(S)$ en una muestra o localidad.

Curvas de abundancia-biomasa (ABC): Las curvas de abundancia-biomasa (ABC) han sido utilizadas para determinar el nivel de alteración o grado de estrés ecológico de las comunidades (Warwick \& Clarke, 2001). Se comparó la disposición de las curvas en un mismo gráfico de abundancia y biomasa de las especies en forma de curvas tipo $k$-dominancia (Lambshead et al., 1983; Warwick, 1986). El modelo asume que en condiciones estables, las especies estrategas tipo $k$, de gran tamaño, longevas, ritmo de crecimiento lento dominan por su biomasa. A medida que en la comunidad se incremente el grado de estrés por perturbaciones de diversa índole, se verá afectada la biomasa de estas especies, tendiendo a disminuir. Por otra parte, empezarán a dominar en abundancia numérica las especies estrategas tipo $r$ (de tallas pequeñas, de rápido crecimiento y vida corta) (Warwick, 1986; Clarke \& Warwick, 2001; Yemane et al., 2005). Las especies se ordenan en el eje $\mathrm{X}$ (en escala logarítmica) en términos de su porcentaje acumulado de abundancia o biomasa, el estadístico $W$ (Warwick \& Clarke, 1994) representa el área de diferencia entre las dos curvas.

$$
W=\Sigma B i-A i S i=1 /[50(S-1)]
$$

donde: $S$ describe el cambio en el número total de especies, $A i$ es la abundancia total en cada muestra $i$ y $B i$ es la biomasa total en cada muestra $i$. Este modelo propone que la curva de biomasa debe encontrarse por encima de la curva de abundancia $(W>0)$; para ser considerada un área sin estrés, moderadamente estresada cuando coincidan las dos curvas con valores próximos a cero de $(W \sim 0)$ y fuertemente estresado, cuando la curva de biomasa quede por debajo de la curva de abundancia $(W<0)$ (Warwick, 1986). Los valores del estadístico $W$ fluctúan entre -1 a 1 . De acuerdo al supuesto del modelo, cuando el valor del estadístico $W$ es negativo representa indicios de perturbación, lo que reflejará grado de estrés, y cuando el valor del estadístico es positivo, predice que no existe perturbación, y por lo tanto, la comunidad no presenta grado de estrés.

Para los cálculos de los índices de diversidad se utilizó el programa estadístico PRIMER v 6 (Clarke \& Gorley, 2001).

\section{RESULTADOS}

Composición de especies: Se obtuvo un total de 32757 individuos, pertenecientes a dos clases, 26 órdenes, 55 familias, 93 géneros y 148 especies; los peces representaron $70.7 \%$ de la FAC (el resto estaba integrado por crustáceos, moluscos y otros). El orden Perciformes fue el más diverso con 18 familias, 25 géneros y 43 especies. Las familias mejor representadas en número de especies fueron: Scianidae con 21, Paralichthydae con 11 y las familias Haemulidae y Serranidae con 10 especies cada una. Las especies más abundantes de mayor constancia y frecuencia de ocurrencia (FO), fueron: Porichthys analis (Hubbs \& Schultz, 1939), Diplectrum macropoma (Günther, 1864), Urobatis halleri (Cooper, 1863), Pomadasys panamensis (Steindachner, 1875), Micropogonias megalops (Gilbert, 1890) y Synodus scituliceps (Jordan \& Gilbert, 1882). Estas fueron parte de las 15 especies en orden de importancia que aportaron el $72 \%$ de la captura total (Cuadro 1), que a su vez formaron parte de las 30 especies más abundantes y frecuentes que aportaron $90 \%$ de la abundancia relativa acumulada.

Índice del valor biológico (IVB): La dominancia numérica estimada a través del IVB mostró que la mayoría de las especies encontradas fueron de hábitat demersal, asociados a fondos blandos (Cuadro 1), Eucinostomus dowii (Gill, 1863) fue la especie más importante en el puntaje de IVB $=480.25$ 
CUADRO 1

Especies que representaron $90 \%$ de la abundancia relativa acumulada e índice de valor biológico IVB en el norte del Golfo de California durante 2010-2011

TABLE 1

List of species representing $90 \%$ of relative accumulated abundance and IVB (biological index value) in the Northern Gulf of California from 2010-2011

\begin{tabular}{|c|c|c|c|c|c|c|c|c|}
\hline Familia & Género-especie & A & B & $\mathrm{AR}$ & $\mathrm{BR}$ & $\mathrm{FO}$ & $\mathrm{FO} \%$ & IVB \\
\hline Batrachoididae & Porichthys analis & 3596 & 124163.75 & 12.74 & 7.55 & 74 & 62.18 & 470 \\
\hline Serranidae & Diplectrum macropoma & 2255 & 87767.1 & 7.99 & 5.34 & 72 & 60.50 & 372 \\
\hline Urolophidae & Urobatis halleri & 1934 & 244705.17 & 6.85 & 14.81 & 80 & 67.22 & 433 \\
\hline Haemulidae & Pomadasys panamensis & 1723 & 70713.5 & 6.10 & 4.30 & 82 & 68.90 & 393 \\
\hline Scianidae & Micropogonias megalops & 1461 & 140768.9 & 5.17 & 8.56 & 68 & 57.14 & 307 \\
\hline Synodontidae & Synodus scituliceps & 1417 & 166109.2 & 5.02 & 10.12 & 75 & 63.02 & 396 \\
\hline Gerridae & Eucinostomus dowii & 1412 & 64372.7 & 5.00 & 3.91 & 44 & 36.97 & 480 \\
\hline Scorpaenidae & Scorpaena sonorae & 1198 & 33171.1 & 4.24 & 2.01 & 74 & 62.18 & 353 \\
\hline Triglidae & Prionotus stephanophrys & 1151 & 48044 & 4.07 & 2.92 & 51 & 42.85 & 232 \\
\hline Paralichthyidae & Etropus crossotus & 906 & 9263.1 & 3.21 & 0.56 & 47 & 39.49 & 173 \\
\hline Haemulidae & Orthopristis reddingi & 883 & 60414.5 & 3.12 & 3.67 & 58 & 48.73 & 216 \\
\hline Mullidae & Pseudupeneus grandisquamis & 713 & 31527.7 & 2.52 & 1.91 & 54 & 45.37 & 208 \\
\hline Synodontidae & Synodus lucioceps & 643 & 53370.9 & 2.27 & 3.24 & 19 & 15.96 & 103 \\
\hline Triglidae & Prionotus birostratus & 573 & 1221.4 & 2.03 & 0.07 & 29 & 24.37 & 70 \\
\hline Serranidae & Paralabrax maculatofasciatus & 571 & 36965.7 & 2.02 & 2.24 & 52 & 43.69 & 136 \\
\hline Gerridae & Eucinostomus currani & 514 & 21247.2 & 1.82 & 1.29 & 17 & 14.28 & 89 \\
\hline Haemulidae & Haemulopsis nitidus & 499 & 22076.6 & 1.76 & 1.34 & 41 & 34.45 & 235 \\
\hline Haemulidae & Haemulopsis elongatus & 473 & 25017.1 & 1.67 & 1.52 & 31 & 26.05 & 137 \\
\hline Cynoglossidae & Symphurus chabanaudi & 425 & 10483.9 & 1.50 & 0.63 & 65 & 54.62 & 179 \\
\hline Paralichthyidae & Syacium ovale & 422 & 7366.4 & 1.49 & 0.44 & 48 & 40.33 & 138 \\
\hline Scianidae & Cynoscion squamipinnis & 417 & 8099.3 & 1.47 & 0.49 & 22 & 18.48 & 107 \\
\hline Paralichthyidae & Citharichthys fragilis & 394 & 4406.6 & 1.39 & 0.26 & 25 & 21.00 & 102 \\
\hline Serranidae & Diplectrum pacificum & 290 & 27122.5 & 1.02 & 1.65 & 62 & 52.10 & 103 \\
\hline Balistidae & Balistes polylepis & 280 & 19876.9 & 0.99 & 1.20 & 52 & 43.69 & 123 \\
\hline Ophidiidae & Lepophidium prorates & 247 & 10491.7 & 0.87 & 0.63 & 48 & 40.33 & 111 \\
\hline Pleuronectidae & Pleuronichthys ocellatus & 241 & 8424.5 & 0.85 & 0.51 & 33 & 27.73 & 73 \\
\hline Haemulidae & Xenistius californiensis & 195 & 10259.6 & 0.69 & 0.62 & 19 & 15.96 & 63 \\
\hline Gobiidae & Bollmannia stigmatura & 193 & 1018.6 & 0.68 & 0.06 & 27 & 22.68 & 98 \\
\hline Paralichthyidae & Citharichthys platophrys & 180 & 2240.4 & 0.63 & 0.13 & 6 & 5.042 & 32 \\
\hline Tetradontidae & Sphoeroides annulatus & 176 & 14449.6 & 0.62 & 0.87 & 43 & 36.13 & 144 \\
\hline
\end{tabular}

$\mathrm{A}=$ Abundancia numérica, $\mathrm{B}=$ biomasa en peso, $\mathrm{AR}=$ abundancia relativa, $\mathrm{BR}=$ biomasa relativa, $\mathrm{FO}=$ frecuencia de ocurrencia, $\mathrm{FO}=$ frecuencia relativa, $\mathrm{IVB}=$ índice valor biológico.

con una abundancia relativa $\mathrm{AR}=5.00 \% \mathrm{y}$ frecuencia de ocurrencia FO $\%=36.97$ sobrepasando las 15 primeras especies en orden de abundancia; mientras que $P$. analis, fue la segunda en importancia, obteniendo IVB $=470$ en 74 lances y ocupando la tercera posición en $\mathrm{FO} \%=62.18$ pero primer lugar en $\mathrm{AR}=12.47$
$\%$. La manta redonda $U$. halleri en tercera posición con IVB $=433.5$ pero segundo lugar en FO $\%=67.22 \%$ y AR $=6.85 \%$, S. scituliceps (Jordan \& Gilbert, 1882) ocupó el cuarto lugar con IVB $=396.5$ con $\mathrm{FO}=75$ lances con $\mathrm{FO}$ $\%=63.02 \%$ y una $\mathrm{AR}=5.02 \%$. En quinta posición según el IVB $=393.7, \mathrm{AR}=6.10 \%$ 
y primer lugar de FO $\%=68.91 \%$, estuvo $P$. panamensis (Steindachner, 1876) constante en 82 muestras. M. megalops (Gilbert, 1890) presenta una alta abundancia numérica, siendo también de las más constantes, ocupa la octava posición en el IVB $=307.25$ con frecuencia de ocurrencia de 68 lances, FO \% $=57.14 \%$ y AR $=5.17 \%$ (Cuadro 1$)$.

Diversidad: Los valores de los índices ecológicos mostraron una comunidad muy diversa para la región del NGC, así mismo variación entre los meses de captura (Fig. 2). Los valores mensuales mostraron promedio $H^{\text {' }}$ $=3.05\left(2.72>H^{\prime}<3.25\right)$ bits/ind, con el valor más alto de $H^{\prime}=3.25 \mathrm{ind} / \mathrm{bit}$ en octubre 2010 , y el valor más bajo en febrero 2010 y enero 2011 , con $H^{\prime}=2.72 \mathrm{ind} /$ bit, seguida de marzo 2010 con $H^{\prime}=2.92 \mathrm{ind} / \mathrm{bit}$. En el resto de los meses se encontraron valores por encima de 3 . El índice de equitatividad J' $=0.71$ mostró una comunidad dominada por algunas especies y no se observaron cambios marcados $(0.66<\mathrm{J}$ ' $>0.81$ ) (Fig. 2). En general, los índices ecológicos presentan tendencia a decrecer conforme avanzó la temporada de pesca.

Curvas comparativas abundancia-biomasa ABC: De acuerdo a los resultados positivos basados en el valor del estadístico $W$,
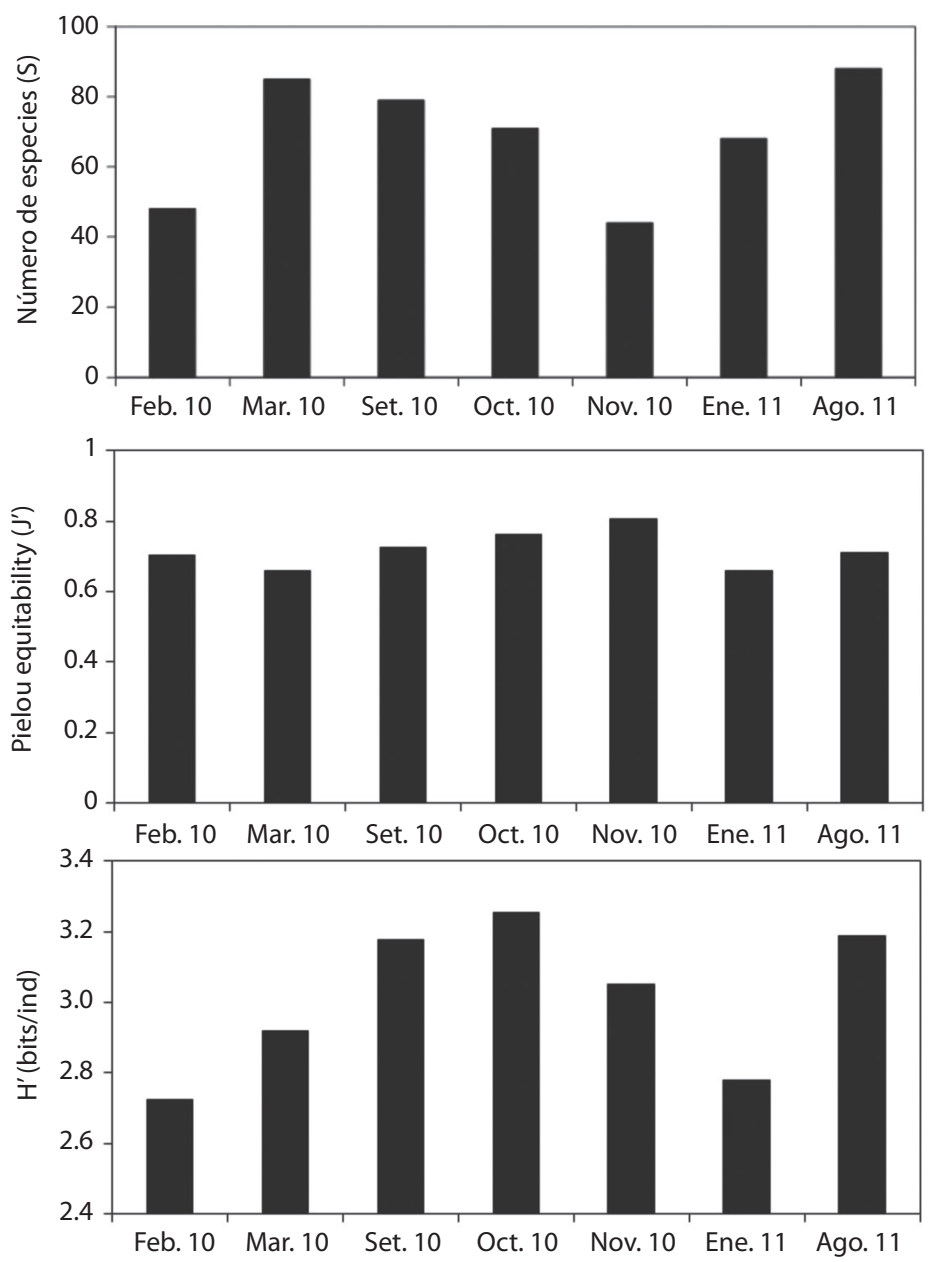

Fig. 2. Índices ecológicos de la comunidad íctica en el norte del Golfo de California durante 2010-2011. Fig. 2. Ecological indexes of the fish community in the Northern Gulf of California from 2010-2011. 
obtenido a través de la comparación de las curvas abundancia-biomasa durante los meses de la temporada de pesca 2010: febrero, octubre y noviembre y agosto 2011, la comunidad no presentó grado de estrés (Fig. 3). Sin embargo, en la misma temporada 2010 durante los meses de marzo y septiembre, el estadístico $W$ mostró un valor negativo, al igual que en enero 2011, por lo que, estos valores negativos identificaron periodos con un grado de estrés de nivel moderado. Confirmado por la comparación entre curvas de abundancia y biomasa (Fig. 4), es decir, la abundancia está por encima de la biomasa debido al efecto provocado por perturbaciones de tipo antropogénico y/o natural. Mientras que en los meses de febrero, octubre y noviembre de la temporada 2010 y agosto 2011 la curva comparativa de la biomasa se encuentra por arriba de la curva de la abundancia, que de acuerdo a los supuestos del método ABC, para estos meses representa una comunidad de peces en buen estado.

\section{DISCUSIÓN}

El número de especies observadas en este estudio se considera robusto, debido al tipo e intensidad de muestras analizadas durante el periodo de pesca. La temporada de pesca de arrastre para camarón en embarcaciones mayores inicia a mediados de septiembre y finaliza en marzo, existiendo un periodo de reposo de seis meses. Este periodo de no actividad pesquera, ayuda en la recuperación de los fondos sometidos al arrastre en los caladeros de pesca. Cabe mencionar que durante la temporada de pesca, varios industriales dueños de las embarcaciones cesan actividades para determinar una "autoveda" en función del decaimiento de las capturas y del precio del camarón en el mercado internacional (López et al., 2010). Aunque, si bien es por medidas económicas, esta determinación se suma a la recuperación natural del sistema ante posibles afectaciones antropogénicas en las comunidades de los fondos blandos. Considerando que, el periodo de veda establecido para esta actividad inicia a principios de abril y finaliza a mediados de septiembre. Adicionado a que solo una fracción de la comunidad de peces demersales es explotada en los caladeros de pesca con fondos blandos muy cercanos a la costa, entre $25-40 \mathrm{~m}$. Lo cual, quizá explique una parte de los resultados, es decir, una comunidad con nivel de estrés de tipo moderado. En este estudio participaron 13 embarcaciones en diferentes tiempos, las muestras que fueron analizadas se obtenían de los caladeros de pesca del NGC, fuera del área de amortiguamiento de la $\mathrm{RB}$, debido a la exclusión de las embarcaciones mayores en el área protegida. El estudio registró 148 especies de peces, mayor a lo encontrado por

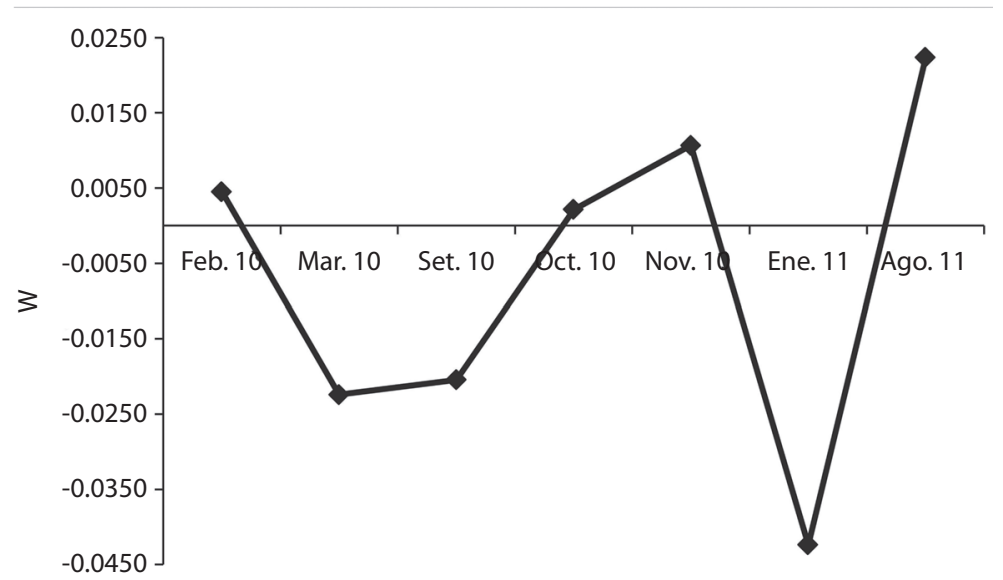

Fig. 3. Valor del estadístico $W$ de las curvas Abundancia Biomasa.

Fig. 3. Statistical value of $\mathrm{W}$ between the curves of Comparative Abundance- Biomass ABC. 

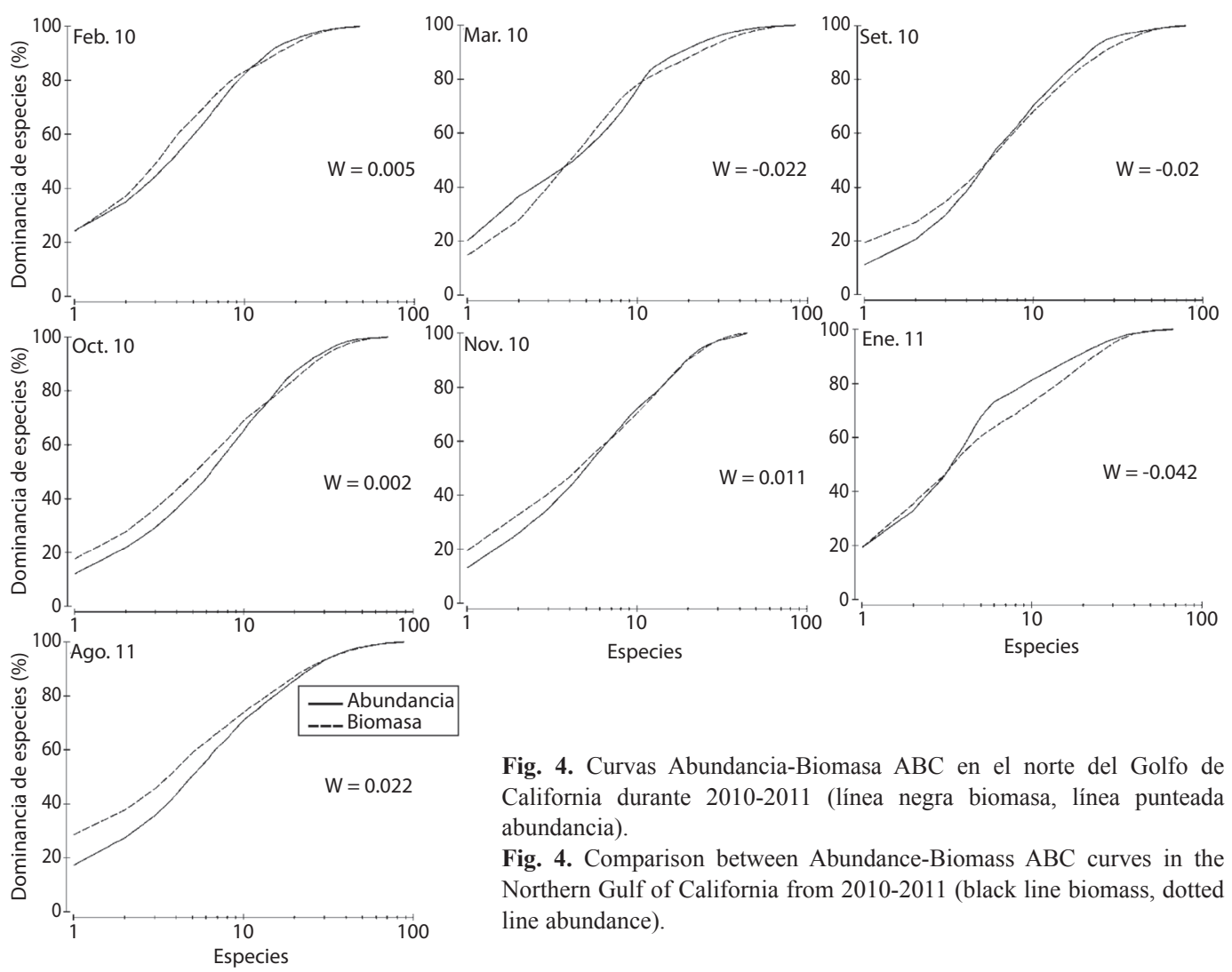

Especies

Fig. 4. Curvas Abundancia-Biomasa $\mathrm{ABC}$ en el norte del Golfo de California durante 2010-2011 (línea negra biomasa, línea punteada abundancia).

Fig. 4. Comparison between Abundance-Biomass $A B C$ curves in the Northern Gulf of California from 2010-2011 (black line biomass, dotted line abundance).

Nava (1994) quien reportó 76 especies de peces provenientes de cruceros comerciales para la misma área de pesca y con lances dentro de lo que hoy es zona de amortiguamiento dentro de la RB. Aunque en dicho estudio, los organismos fueron registrados y agrupados a nivel de familia y género, por lo que hay una pérdida de información a nivel de especies y se descarta comparar en su totalidad.

Hasting y Findley (2007), registraron 260 especies de peces provenientes de varias fuentes realizadas en zonas libres para la pesca y en la zona de amortiguamiento de la RB, por lo que una gran proporción de especies no fueron observadas en este estudio, debido a la restricción de zonas de pesca para las embarcaciones mayores.

La riqueza fue mayor a la registrada por Calderón (2011) en el norte Golfo de California, donde enlista 134 especies de peces (70
$\%$ de las especies totales de la FAC), lo que coincide con el presente estudio. Cabe mencionar que en los resultados de su estudio también fue considerada el área de amortiguamiento de la RB.

Por lo anterior, se cuenta con registros de las mismas especies (Nava 1994) antes del decreto de la reserva (1995) y en la actualidad, lo que permite afirmar que las especies registradas en 1993-1994 siguen siendo las mismas especies componentes de la FAC en el NGC. Especies como S. scituliceps, U. halleri y $P$. panamensis fueron registradas por Nava (1994) y son especies consideradas de amplia distribución latitudinal y batimétrica del Pacífico Oriental Tropical (POT) (Robertson \& Allen, 2002; Castro, Balart, \& Arvizu, 1995). Estas especies continúan formando parte de las 30 especies más abundantes de la FAC dentro del Golfo de California (Pérez \& Findley, 1985; 
Valenzuela, Gracia, \& Herrera, 1988; López et al., 2007; Madrid, Amezcua, \& Morales, 2007; Rábago et al., 2011). Existen además de las especies anteriores, otras muy abundantes que se encuentran restringidas al NGC, entre las que están $M$. megalops, $S$. sonorae y $P$. analis (Nava, 1994; Cudney \& Turk, 1998).

La amplia distribución de algunas de las especies de peces presentes se debe a su adaptación biológica a diversos hábitats y a condiciones ambientales en la zona costera del Golfo de California (Hernández \& Solís, 2005; López et al., 2010) considerado como uno de los ecosistemas marinos más ricos del mundo (Cudney \& Turk, 1998), por lo que sostiene grandes cadenas alimenticias derivado del gran potencial alimenticio autotrófico. Lo anterior se reflejó en los índices de diversidad de $\mathrm{H}^{\prime}=$ 3.25 bits/ind en la estructura de la comunidad de peces demersales, similares a los valores $\mathrm{H}^{\prime}=3.39$ bits/ind reportados en el estudio de Calderón (2011). Aunque en otras áreas del sur del Golfo de California, en un estudio reciente, particularmente en el sur de Sinaloa y norte de Nayarit (Nieto, Zetina, Arreguín, Palacios, \& Jordan, 2013) se reportaron valores de $H^{\prime}=4.1$ bits/ind muy superiores a nuestros resultados, y a lo reportado en el estudio de Godínez y González (1999) sobre macroinvertebrados bentónicos en las costas de Jalisco y Colima $\left(\mathrm{H}^{\prime}=\right.$ 2.39) bits/ind en experimentos efectuados en fondos blandos.

Por otro lado, el análisis comparativo entre curvas abundancia biomasa sugiere la presencia de especies pequeñas dominantes, como: $P$. analis, U. halleri, P. panamensis, Diplectrum macropoma, S. sonorae, Syacium ovale y Prionotus stephanophrys las cuales explican las diferencias entre las curvas $\mathrm{ABC}$ dentro de la comunidad (Clarke \& Warwick, 2001), lo que coincide con los resultados en el estadístico $W$ con valores negativos durante los meses de marzo $(W=-0.022)$ y septiembre $2010(W=$ -0.02), y enero 2011 ( $W=-0.042)$, al mostrar un grado moderado de estrés ecológico. El origen de dichos cambios aún no tiene concenso entre los autores: algunos señalan que podrían ser derivados de las perturbaciones generadas por los efectos de la pesca de arrastre (Kaiser, Collie, Hall, Jennings, \& Poiner, 2002; Godínez, 2003), porque es considerada la actividad de mayor impacto sobre fondos marinos; esta provoca no sólo mortalidad directa de especies no objetivo, sino también cambios en la diversidad de especies, con efectos indirectos sobre la red trófica por pérdida de depredadores, y destrucción y modificación de hábitat, así como resuspensión de material de fondo provocada por el arrastre continuo (Kaiser et al., 2002; Thrush \& Dayton, 2002). En los meses donde el nivel de estrés es bajo, Jouffre y Inejih (2005) mencionan en su estudio que la fuerte resistencia del ecosistema puede ser lo que causa esta discrepancia. Aunque actualmente los investigadores no llegan a un acuerdo respecto al valor negativo del estadístico $W$ para definir una comunidad sobreexplotada (Jouffre \& Inejih, 2005), el método logra identificar cambios temporales a escala estacional en las comunidades ícticas (Pérez, 2011).

No obstante lo anterior, es necesario hacer algunos alcances teóricos respecto a las curvas $\mathrm{ABC}$. En primer lugar, pese a que son una herramienta bastante utilizada en la literatura, éstas han sido objeto de una serie de críticas inicialmente por Beukema (1988) quien encontró inconsistencias del método verificadas cuando se presenta una gran dominancia numérica ecológica de especies o formas de pequeño tamaño relativo y de vida corta relacionadas con periodos de reclutamiento. En su estudio sobre contaminación en sedimentos marinos, Burd, Nemee y Brinkhust (1990) encontraron dos limitantes en el método curvas $\mathrm{ABC}$ : por un lado asumen que el conjunto faunístico analizado estaba inicialmente en una condición estable o de equilibrio, condición que raramente es efectiva o real en hábitats que son controlados ambientalmente, y la otra limitante refiere a que las estaciones moderadamente contaminadas, muestran análisis muy interesantes, pero con resultados ambiguos.

Godínez (2003) menciona que los cambios espaciales como la batimetría, también pueden ser considerados como perturbación, y que podría ser erróneo concluir que la pesca 
es la que produce el efecto negativo, en donde la dominancia de especies pequeñas puede interpretarse equivocadamente como un disturbio en la comunidad. Thrush, Hewit, Gibbs, Lundquist y Norkko (2006) sugieren que la comunidad puede responder a niveles de estrés de acuerdo a la intensidad del disturbio, que podrían estar siendo provocados por factores independientes de la pesca, como cambios estacionales en el reclutamiento, o cambios en los ciclos de vida de las especies. Sin embargo, en el estudio de Nieto (2010) sobre la aplicación del método $\mathrm{ABC}$, llega a la conclusión que la pesca de arrastre no fue altamente significativa para concluir que el nivel de deterioro sobre las comunidades de fondos blandos sea atribuible a la pesca.

Aunque en el trabajo de Pérez (2011) que comparó área de arrastre con otra de no perturbación, particularmente para macroinvertebrados de la FAC concluye que las curvas $\mathrm{ABC}$ detectaron un alto grado de estrés en las zonas donde operó la flota de arrastre camaronera, ya que la abundancia estuvo siempre por encima de la biomasa, parcialmente diferente a lo encontrado en el NGC.

De lo anterior se desprende que, si bien los resultados de las curvas comparativas $\mathrm{ABC}$ denotan comunidades en buen estado y moderadamente alteradas, lo que coincide con los índices bioecológicos, éstos deben realizarse continuamente como sugirieron Aderlini y Wear (1992) para suministrar una evaluación más exacta ante posibles disturbios físicos, biológicos o de otra naturaleza. Así mismo, deberán ser combinados con información ambiental y esfuerzo pesquero, para poder asociar a uno $\mathrm{u}$ otro como causa principal, sobre todo considerando lo altamente dinámico que es el NGC (Kurczyn, Beier, Lavin, \& Chaigneau, 2012).

Así mismo, la carencia de estudios similares para el área de estudio dificulta la comparación de los resultados aquí presentados, limitando la deducción sobre la riqueza y la abundancia de especies. Por otro lado no se identifican cambios en la composición y biomasas de las especies más abundantes y frecuentes anteriormente registradas. Sin embargo, esas
30 especies siguen soportando presión pesquera principalmente en los estratos de profundidad 25-40 m, por lo que es necesario realizar estudios directos sobre estas poblaciones.

De acuerdo a la pesca de arrastre efectuada por barcos camaroneros en el Norte Golfo de California, como factor detonante para evidenciar cambios en la estructura de la comunidad de peces y en la composición íctica estimadas por el método curvas comparativas abundancia biomasa $\mathrm{ABC}$ a través de su estadístico $\mathrm{W}$, mismos que mostraron valores negativos, se presentó estrés moderado en la comunidad, aunque ambiguo y no explicado totalmente por la actividad de pesca. Las variaciones en la diversidad y abundancias son explicadas por el patrón espacial y temporal de las especies, asociada a la estabilidad y resiliencia del ecosistema.

Los resultados en general fueron explicados por el comportamiento que sigue el periodo de captura y la forma en que operan las embarcaciones.

Es crucial estimar la composición de las especies que forman parte de la (FAC), para identificar aquellas especies que están siendo afectadas en su contribución a los atributos comunitarios como la riqueza, diversidad y abundancias relativas debido a que se encuentran ligados directamente con el funcionamiento del ecosistema.

Es necesario continuar con los análisis espacio temporales entre los diferentes caladeros de pesca del área de estudio, con base en la información de cruceros de investigación y viajes comerciales, hasta lograr una serie robusta de listados ictiofaunísticos, para su manejo, y poder detectar niveles de perturbación en combinación con otros métodos de análisis multifactoriales para la evaluación de comunidades biológicas, que permitan evidenciar cambios en la estructura comunitaria.

Se recomienda efectuar un análisis de esfuerzo pesquero de las especies comerciales y relacionarlo con información biológica de las poblaciones de peces sujetas a la pesca objetivo, integrando estudios de dinámica poblacional para identificar cambios en las medidas 
de longitud, talla de primera madurez sexual, patrón de reclutamiento, etc.

\section{AGRADECIMIENTOS}

Este trabajo fue financiado por el proyecto 26-2009-1356 de la Fundación PRODUCE Sonora. Se agradece al proyecto EP1 y al laboratorio de Pesquerías de la Unidad Guaymas del Centro de Investigaciones Biológicas del Noroeste, S.C, en particular a Rufino Morales Azpeitia. Se agradece a Diana Dorantes por la edición del inglés. Se agradece al INAPESCA y a los dueños de los barcos por facilitar la toma de muestras a bordo.

\section{RESUMEN}

La pesca de arrastre ha sido considerada como una actividad que impacta y modifica hábitats, provoca cambios en la composición y abundancias de las especies y altera la estructura y funcionamiento del ecosistema, generando finalmente una pérdida de la biodiversidad. La parte norte del Golfo de California ha sido considerada una zona mega diversa de alto endemismo y es motivo de interés creciente por la comunidad científica internacional. Con la finalidad de evaluar potenciales cambios en la comunidad de peces componentes de la fauna de acompañamiento del camarón (FAC), durante 2010-2011 se analizaron 119 arrastres de 13 embarcaciones camaroneras con base en Puerto Peñasco, a partir de 14 viajes de pesca comercial realizados a 9-90 m. Una muestra aleatoria de $20 \mathrm{~kg}$ fue obtenida de cada arrastre y analizada en el laboratorio para la composición de las especies. Adicional al Índice de Valor Biológico (IVB), diversidad de Shannon (H'), equitatividad de Pielou (J') fueron estimadas las curvas comparativas Abundancia-Biomasa (ABC). Eucinostomus dowii fue la mayor en IVB $=480.25$, Porichthys analis la de mayor abundancia relativa y Pomadasys panamensis presentó la mayor frecuencia de ocurrencia. Los valores mensuales promedio en la diversidad $H^{\prime}=3.05\left(2.72>H^{\prime}\right.$ $<3.25)$ y J' $=0.71\left(0.66<\mathrm{J}^{\prime}>0.81\right)$ presentaron tendencia a decrecer conforme avanza la temporada de pesca. El comparativo curvas abundancia-biomasa $(\mathrm{ABC})$ y el valor del estadístico $W$, evidenciaron a marzo $(\mathrm{W}=-0.022)$ y septiembre $(\mathrm{W}=-0.02) 2010$ y enero $2011(\mathrm{~W}=-0.042)$ con niveles de estrés moderado. En conclusión, la región norte del Golfo de California presentó una comunidad bien estructurada con grado de estrés moderado, aunque no explicado totalmente por la pesca.

Palabras clave: norte del Golfo de California, pesca incidental de arrastre, curvas ABC, comunidad, estrés.

\section{REFERENCIAS}

Anderlini, V. C., \& Wear, R. G. (1992). The effect of sewage and natural seasonal disturbances on benthic macrofaunal communities in Fitzroy Bay, Wellington, New Zealand. Marine Pollution Bulletin, 24, 21-26.

Beukema, J. J. (1988). An evaluation of the ABC-method (abundance/biomass comparison) as applied to macrozoobenthic communities living on tidal flats in the Dutch Wadden Sea. Marine Biology, 99, 425-433.

Box, E. G., Stuart, J. H., \& Hunter, W. G. (2008). Estadística para investigadores. Diseño, innovación y descubrimiento. Barcelona, España: Wiley.

Burd, B. J., Nemee, A., \& Brinkhust, R. O. (1990). “The Development y application of analytical method in benthic marine infaunal studies" Advances in Marine Biology, 26, 170-247.

Caddy, F. J. (2007). Marine habitat and Cover: Their importance for productive coastal fishery resources. Paris, France: UNESCO.

Calderón, A. A. (2011). Evaluación piloto de los impactos potenciales de las redes de arrastre sobre el ecosistema del Alto Golfo de California durante la temporada de pesca 2010-2011 (Margarita Caso coord., Convenio N NE/A1-038/2010). D.F., México: Centro de Investigación Científica y de Educación Superior de Ensenada, Baja California.

Castro-Aguirre, J. L. (1978). Catálogo sistemático de los peces marinos que penetran a las aguas continentales de México, con aspectos zoogeográficos y ecológicos. D.F., México: Depto. de Pesca. I.N.P.

Castro, A. J. L., Balart, P. E. F., \& Arvizu, M. J. (1995). Contribución al conocimiento del origen y distribución de la ictiofauna del Golfo de California, México. Hidrobiológica, 5, 57-58.

Castro-Aguirre, J. L. \& Espinosa-Pérez, H. (1996). Listados faunísticos de México. Catálogo sistemático de las rayas y especies afines de México (Chondrichthyes: Elasmobranchii: Rajiformes: Batoideiomorpha) (Vol. VII, pp. 75). D.F., México: Instituto de Biología, Universidad Nacional Autónoma de México.

Clarke, K. R., \& Gorley, R. N. (2001). PRIMER (Plymouth Routines In Multivariate Ecological Research) v5: User Manual/Tutorial PRIMER-E Plymouth (pp. 91). U.K.: Plymouth Marine Laboratory.

Clarke, K. R., \& Warwick, R. M. (2001). Change in marine communities: An Approach to Statistical Analysis and interpretation. UK: E-primer-E Ltd, Plymouth Marine Laboratory.

Compagno, L. J. V. (1984). FAO species catalogue. Sharks of the world. An annotated and illustrated catalogue of sharks species known to date, Hexanchiformes to Lamniformes. FAO Fisheries Synopsis, 4(125), $1-249$. 
Cudney, B. R. \& Turk, B. P. J. (1998). Pescando entre mareas del Alto Golfo de California: una guía sobre la pesca artesanal, su gente, y sus propuestas de manejo. Puerto Peñasco, Sonora, México: Centre Intercultural de Estudios de Desiertos y Océanos (CEDO) A.C.

Curran, H. W. (1942). A systematic revision of the gerreid tishes referred to the genus Eucinostomus with a discussion of their distribution and speciation (Doctoral thesis). University of Michigan, USA.

Dernie, K. M., Kaiser, M. J., Richardson, E. A., \& Warwick, R. M. (2003). Recovery of soft bottom sediment communities and habitats following physical disturbance. Journal of Experimental Marine Biology and Ecology, 285-286, 415-434.

DOF. (1993). Decreto por el que se declara área natural protegida con el carácter de la Reserva de la Biosfera, la región conocida como Alto Golfo de California y Delta del Río Colorado, ubicada en aguas del Golfo de California y los municipios de Mexicali, BC., de Puerto Peñasco y San Luis Río Colorado, Son. México.

Fischer, W., Krupp, F., Schneider, W., Sommer, C., Carpenter, K. E., \& Niem, V. H. (Eds.). (1995). Guía FAO para la identificación de especies para los fines de pesca (Vol. II-III). Roma: FAO.

Ginsburg, I. (1958). Flounders of the genus Paralichthys and related genera in american waters. Fishery Bulletin. United States Fish and Wildlife Service, 52, 267-351.

Godínez, D. E. \& González, S. G. (1999). Diversidad de macroinvertebrados de fondos blandos de la plataforma continental de Jalisco y Colima, México. Ciencias Marinas, 25(4), 609-627.

Godínez, D. E. (2003). Ecología de las asociaciones de macroinvertebrados bentónicos de fondos blandos del Pacífico central mexicano (Tesis de doctorado). Universidad de La Coruña, España.

Hastings, P. A., \& Findley, L. T. (2007). Marine fishes of the biosphere reserve, Northern Gulf of California. In R. Felger, \& W. Broyles (Eds.), Dry Borders: Great Natural Areas of the Gran Desierto and Upper Gulf of California (pp. 364-382). Salt Lake City, Utah: University of Utah Press.

Hernández, A. P., \& Weiss, S. V. (2005). Seasonal Variations of the Spionida (Palpata: Canalipalpata) in the Sublittoral Zone of the Gulf of California. Marine Ecology, 26, 273-285.

Jennings, S., \& Reynolds, J. G. (2000). Impact fishing on diversity: from pattern to process. In M. Kaiser, \& S. J. de Groot (Eds.), Effect of fishing on Non-Target Species and Habitats (pp. 235-248). USA: Blackwell Science.
Jordan, D. S., \& Evermann, B. W. (1896-1900). The fishes of North and Middle America. Bulletin Natural Museum, (47), 1-3313.

Jouffre, D., \& Inejih, C. (2005). Assessing the impact of fisheries on demersal fish assemblages of the Mauritanian continental shelf, 1987-1999, using dominance curves. Journal Marine Science, 62, 380-383.

Kaiser, M., Collie, S., Hall, S., Jenning, S., \& Poiner, I. (2002). Modification of marine habitats by trawling activities: prognosis and solutions. Fish and Fisheries, 3(2), 114-136.

Kurczyn, R. J. A., Beier, E. J., Lavin, P. M. F., \& Chaigneau, A. (2012). Mesoscale eddies in the northeastern Pacific tropical-subtropical transition zone: Statistical characterization from satellite altimetry. Geophysical Research. Oceans, 117(C10021) doi: 10.1029/2012JC007970

Lambshead, P. J. D., Plat, H. M., \& Shaud K. M. (1983). The detection of differences among assemblages of marine benthic species based on an assessment of dominance and diversity. Journal of Natural Story, $17,859-847$

López, M. J., Hernández, V. S., Herrera, V. E., Rábago, Q. C. H., \& Morales, A. R. (2007). Efectos ecológicos de la pesca de arrastre de camarón en el Golfo de California. Estado del arte del desarrollo Tecnológico de las Artes de Pesca. In CEDRSSA (Ed.), $L a$ situación del sector pesquero en México (pp-14-47). D.F., México: CEDRSSA y Cámara de diputados LX Legislatura.

López, M. J., Herrera, V. E., Rodríguez, R. J., \& Hernández, V. S. (2010). Peces de la fauna de acompañamiento de la pesca industrial del camarón del Golfo de California, México. Revista de Biología Tropical, 58(3), 925-942.

López, M. J., Rodríguez, R. J., Hernández, S. N. Y., \& Herrera, V. E. (2011). Population parameters of the Pacific flagfin mojarra Eucinostomus currani (Perciformes: Gerreidae) captured by the shrimp trawling fishery in the Gulf of California. Revista de Biologia Tropical, 59, 887-897.

Love, M. S., Yoklavich, M., \& Thorsteinson, L. (2002) Rockfishes of the Northeast Pacific. Berkeley, CA: University of California Press.

Loya, S. D. \& Escofet, H. A. (1990). Aportación al cálculo del índice del Valor Biológico (Sanders, 1960). Ciencias Marinas, 16, 97-115.

Lluch, C. S. E. \& Arias, A. J. P. (2000). Sobre la importancia de considerar la existencia de actividad biológica para la regionalización del océano: el caso del Golfo de California. In D. Lluch-Belda, S. Lluch-Cota, J. Elorduy, \& G. Ponce-Díaz (Eds.), Centros de actividad biológica del Pacífico Mexicano (pp. 255-263). México: CIBNOR-CICIMAR-CONACYT. 
Madrid, V. J., Amezcua, F., \& Morales, B. E. (2007). An assessment approach to estimate biomass of fish communities from bycatch data in a tropical shrimp-trawl fishery. Fisheries Research, 83, 81-89.

Márquez, F. J. (2002). The artisanal ray fishery in the Gulf of California: development, fisheries research, and management issues. IUCN Shark Specialist Group Shark News, 14, 1-5.

Miller, D. J., \& Lea, R. N. (1972). Guide to the coastal marine fishes of California. California, USA: Department Fish and Game, Fisheries Bulletin.

Morales, A. R. (2011). Variación espacio temporal de la relación camarón: FAC en la pesquería de camarón del Golfo de California y su uso como medida de manejo. Implicaciones socioeconómicas (Tesis de doctorado). Universidad Autónoma de Nayarit, México.

McPhail, J. D. (1958). Key to the croakers (Sciaenidae) of the eastern Pacific (Vol. 2, pp. 1-20). Vancouver, Canada, University of British Columbia, Institute of Fisheries Museum Contrubution.

Myers, R. A., \& Worm, B. (2003). Rapid worldwide depletion of predatory fish communities. Nature, 423, 280-283.

Nava, R. J. M. (1994). Impactos, a corto y largo plazo, en la diversidad y otras características ecológicas de la comunidad béntico-demersal capturada por la pesquería de camarón en el norte del alto Golfo de California, México (Tesis de maestría). Instituto Tecnológico y de Estudios Superiores de Monterrey, Guaymas, México.

Nelson, E., Ramírez, E. M., Arenas, C. B., Jacquemine, P., \& Prado, S. N., (1980). Evaluación de los recursos demersales accesibles a redes de arrastre de fondo del Golfo de California (Mar de Cortez), México, durante 1979. México: Programa de Investigación y DAESARROLlO Pesquero Integrado, Programa de las Naciones Unidas para el Desarrollo.

Nieto, N. J. T. (2010). Estructura y organización de la ictiofauna de fondos blandos del sur de Sinaloa: análisis ecológico y topología de taxa (Tesis de doctorado). CICIMAR-IPN, La Paz, B.C.S., México.

Nieto, N. J. T., Zetina, M. R., Arreguín, F. S., Palacios, S. D. S., \& Jordan, F. (2013). Changes in the fish bycatch during the shrimp fishing season along the Eastern coast the mouth of the Gulf of California. Journal of Applied Ichthyology, 1-7. doi: 10.1111/ jai 12160

Norman, J. R. (1934). A systematic monograph of the flat fishes (Heterostomata). Psettedidae, Bothidae, Pleuronectidae (Vol. 1, pp. 1-459). London, U.K.: Trustees of the British Museum (Nat. Hist.)

Pérez, F. D. (2011). Incidencia de la pesca industrial del camarón sobre los macroinvertebrados en el Caribe
Colombiano (Tesis de maestría). Universidad Nacional de Colombia, Sede Bogotá; Centro de Estudios en Ciencias del Mar (CECIMAR), Sede Caribe; Convenio Universidad Nacional de Colombia, INVEMAR Santa Marta, D.T.C.H.

Pérez, M. J. \& Findley, L. T. (1985). Evaluación de la ictiofauna acompañante del camarón comercial capturado en las costas de Sonora y norte de Sinaloa. In A. Yáñez-Arancibia (Ed.), Recursos pesqueros potenciales de México: La pesca acompañante del camarón (pp. 201-254). D.F., México: Prog. Univ. de Alimentos, Instituto de Ciencias del Mar y Limnología, Instituto Nacional de la Pesca, UNAM.

Pielou, E. C. (1966). The measurement of diversity in different types of biological collections. Journal of Theorical Biology, 13, 131-144.

Rábago, Q. C. H., López, M. J., Valdez, H. J. E., \& Nevárez, M. M. O. (2011). Distribución latitudinal y batimétrica de las especies más abundantes y frecuentes en la fauna acompañante del camarón del Golfo de California, México. Revista de Biología Tropical, 59(1), 255-267.

Robertson, D. R., \& Allen, G. R. (2002). Shore fishes of the Tropical Eastern Pacific: an information system. Balboa, Panama: Smithsonian Tropical Research Institute.

Rodríguez, R. J., Abitia, C. L. A., Galván, F., \& Chávez, R. H. (1994). Composición, abundancia y riqueza específica de la ictiofauna de Bahía Concepción, Baja California Sur, México. Ciencias Marinas, 20 (3), 321-350.

Rosenblatt, R. H., \& Johnson, G. D. (1974). Two new species of sea basses of the genus Diplectrum, with a key to the Pacific species. Fish and Game, 60, 178-191.

Sanders, H. L. (1960). Benthic studies in Buzzards Bay. III. The structure of the soft-bottom community. Limnology and Oceanography 5, 138-153.

Shannon, E. C., \& Weaver, W. (1963). The mathematical theory of communication. Illinois, USA: University of Illinois.

Thrush, S., \& Dayton, P. (2002). Disturbance to marine benthic habitats by trawling and dredging. Implications for marine biodiversity. Annual Review of Ecology and Systematics, 33, 449-473.

Thrush, S. F., Hewitt, J. E., Gibbs, M., Lundquist, C., \& Norkko, A. (2006). Functional role of large organisms in intertidal communities: community effects and ecosystem function. Ecosystems, 9, 1029-1040.

Valenzuela, T. M., Herrera, E. V., \&. Gracia, S. N. (1988). Evaluación de la fauna que acompaña al camarón en arrastres comerciales de las costas de Sonora y Sinaloa, México (Tesis de licenciatura). Universidad Autónoma de Sinaola, Mazatlán, Sinaloa. 
Walker, H. J., \& Rosenblatt, R. H. (1988). Pacific Toadfishes of the Genus Porichthys (Batrachoididae) with Descriptions of Three New Species. Copeia, 4, 887-904.

Warwick, R. M. (1986). A new method for detecting pollution effects on marine macrobenthic communities. Marine Biology, 92, 557-562.

Warwick, R. M., \& Clarke, K. R. (1994). Relearning the $\mathrm{ABC}$ : taxonomic changes and abundance/biomass relationships in disturbed benthic communities. Marine Biology, 118, 739-744.
Warwick, R. M., \& Clarke, K. R. (2001). Practical measures of marine biodiversity based on relatedness of species. Oceanography and Marine BiologyAn Annual Review, 39, 207-231.

Yemane, D., Field, J. G., \& Leslie, R. W. (2005). Exploring the effects of fishing on fish assemblages using Abundance Biomass Comparison (ABC) curves. ICES Journal of Marine Science, 62, 374-379.

Zahuranec, J. B. (1967). The Gerreid fishes of the genus Eucinostomus in the Eastern Pacific (Master thesis). University of California, San Diego. 